\title{
WEAKLY ALMOST PERIODIC FLOWS
}

\author{
R. ELLIS AND M. NERURKAR
}

\begin{abstract}
The notion of the enveloping semigroup of a flow is applied to some situations in ergodic theory. In particular, weakly almost periodic functions on groups are studied and Moore's ergodic theorem is proved.
\end{abstract}

\section{INTRODUCTION}

This paper originated in the process of studying a notion of enveloping semigroups in ergodic theory. Enveloping semigroups have played a very crucial role in topological dynamics. Analogous notions and ideas in ergodic theory helped us obtain a simpler proof of C. Moore's ergodicity theorem. A close examination of our proof showed that the underlying flow on the enveloping semigroup was weakly almost periodic and many arguments in the proof were actually consequences of weak almost periodicity. This paper deals with the notion of weak almost periodicity.

$\S I$ discusses some facts about semigroups which we will need later on. In §II we derive a number of dynamical consequences of weak almost periodicity. Applications to the Ryll Nardzewski theorem and ergodic theory are given in §III. Also, we give characterizations of ergodic theoretic notions of mixing and rigidity. In $§ I V$, using the techniques developed so far, we obtain a generalization of a theorem of W. Veech.

\section{DYNAMICAL SYSTEMS AND SEMIGROUPS}

A flow is a pair $(X, T)$, where $X$ is a compact Hausdorff space and $T$ is a locally compact Hausdorff topological group acting on $X$, on the right and the action $(x, t) \rightarrow x \cdot t$ is continuous. Let $\pi_{t}(x)=x \cdot t, x \in X, t \in T$ and let $E(X)=\left\{\overline{\left.\pi_{t} \mid t \in T\right\}}\right.$, where the closure is in the product topology on $X^{X}$. Clearly $E(X)$ has a natural semigroup structure and a natural right $T$ action. For $p \in E(X)$, let $L_{p}(q)=p q$ and $R_{p}(q)=q p, q \in E(X)$. In general $L_{p}$ as a map from $E(X)$ to itself is continuous, but $R_{p}$ is not so. If

Received by the editors February 10, 1988.

1980 Mathematics Subject Classification (1985 Revision). Primary 54H20, 28D15; Secondary $22 \mathrm{E} 46$.

Research by $\mathbf{R}$. Ellis is partially supported by National Science Foundation grant 8701857 . M. Nerurkar wishes to thank the University of Maryland at College Park, where part of this work was done. 
$t \in T$ then $R_{t}$ is continuous and in fact $(E(X), T)$ is itself a flow, called the enveloping semigroup of the flow $(X, T)$ (see [3]). We will be identifying $t$ with $\pi_{t} \in E(X)$ and hence will be thinking of $T$ as a subset of $E(X)$. Given a net $t_{\alpha} \in T, t_{\alpha} \rightarrow p, p \in E(X)$, will mean that $x \cdot t_{\alpha}$ converges to $x p$ for each $x \in X$. (Note that the image of a point $x$ under the map $p$ will be denoted by $x p$.

In this way compact semigroups arise naturally from flows. Hence study of such semigroups is of importance in analyzing the dynamics of the flow. In the following proposition we will summarize some general results about certain kinds of semigroups. We will be mainly interested in applying these results to the enveloping semigroups (arising from flows) all of whose elements are continuous maps.

First we recall that given a general semigroup $E$, a right (left) ideal $I$ is a subset of $E$ such that if $p \in I$ and $q \in E$ then $p q \in I \quad(q p \in I)$. A minimal right (left) ideal is a right (left) ideal which does not contain a proper right (left) ideal.

Proposition I.1 [4]. Let $E$ be a semigroup with compact $T_{1}$ topology such that the right and left multiplications are continuous. Let I be a minimal right (left) ideal in $E$ and $J$ be the set of idempotents in $I$; then

(1) The set $J$ is not empty.

(2) If $v \in J, p \in I$ then $v p=p$ ( $p v=p$ for the left ideals).

(3) If $v \in J$ then I $v$ is a subgroup with identity $v$ ( $v$ I for the left ideals).

(4) $(I v \mid v \in J)$ is a partition of $I((v I \mid v \in J)$ for left ideals).

A flow is said to be almost periodic if the family of maps $\left\{\pi_{l} \mid t \in T\right\}$ is equicontinuous. A pair of points $(x, y), x, y \in X$ is proximal if for some net $t_{\alpha} \in T, x \cdot t_{\alpha}$ and $y \cdot t_{\alpha}$ converge to the same point. A flow is distal if it has no nontrivial proximal pairs. It is well known that the flow $(X, T)$ is almost periodic iff $E(X)$ is a compact topological group and the elements of $E(X)$ are continuous maps. Also $(X, T)$ is distal iff $E(X)$ is a group (see [4]). Based on the work of Troillac [10] the following theorem due to J. Auslander is a sharpening of this result, (see [1]).

Theorem I.2. Let $(X, T)$ be a minimal flow. If each $p \in E(X)$ is continuous, then $(X, T)$ is almost periodic.

\section{WEAKLY ALMOST PERIODIC FLOWS}

Let $(X, T)$ be a flow. Then $C(X)$ will denote the space of all continuous complex-valued maps on $X$. Given $f \in C(X)$ and $p \in E(X)$, set $\left({ }_{p} f\right)(x)=$ $f(x p), \forall x \in X$. A function $f \in C(X)$ is said to be weakly almost periodic (w.a.p.) iff $\left({ }_{t} f \mid t \in T\right)$ is relatively compact in the weak topology on $C(X)$. The following is a theorem of Grothendieck (see [6]). 
Theorem II.1. Let $(X, T)$ be a flow and $f \in C(X)$. Then $f$ is w.a.p. iff $\left({ }_{t} f \mid t \in T\right)$ is relatively compact in the topology of pointwise convergence on $C(X)$.

A flow $(X, T)$ is w.a.p. iff each $f \in C(X)$ is w.a.p..

Proposition II.2. A flow $(X, T)$ is w.a.p. iff each element of $E(X)$ is continuous. Proof. Let $(X, T)$ be w.a.p. and $p \in E(X)$. To show that $x \rightarrow x p$ is continuous, it is enough to verify that for any $f \in C(X)$ the map ${ }_{p} f$ is continuous. Let $t_{\alpha} \rightarrow p$. Since $f$ is w.a.p., without loss of generality we may assume that ${ }_{t} f \rightarrow g$, for some $g$ in $C(X)$, where the convergence is pointwise. Thus,

$$
g(x)=\lim \left({ }_{t} f\right)(x)=\lim f(x \cdot t)=f(x p)=\left({ }_{p} f\right)(x) .
$$

Conversely, let each $p \in E(X)$ be continuous. Let $f \in C(X)$, let $\phi(p)=\left({ }_{p} f\right)$, $\forall p \in E(X)$. Note that if $x_{\alpha} \rightarrow x$, then

$$
\lim \phi(p)\left(x_{\alpha}\right)=\lim \left({ }_{p} f\right)\left(x_{\alpha}\right)=\lim f\left(x_{\alpha} p\right)=f(x p) .
$$

Thus $\phi(p) \in C(X)$. Now we claim that the map $\phi: E(X) \rightarrow C(X)$ is continuous (here $C(X)$ has the topology of pointwise convergence). To see this let $p_{\alpha} \rightarrow p, p_{\alpha}, p \in E(X)$ and $x \in X$. Then

$$
\lim \phi\left(p_{\alpha}\right) x=\lim f\left(x p_{\alpha}\right)=f(x p)=\phi(p) x .
$$

Thus the image of $\phi$ is compact and it contains the set $\left({ }_{t} f \mid t \in T\right)$. Now Grothendieck's theorem implies that $f$ is w.a.p.

Remark II.3. (i) Proposition II.2 shows that products and factors of w.a.p. flows are again w.a.p.

(ii) Also note that if $(X, T)$ is w.a.p. and $S$ is a subgroup of $T$ then $(X, S)$ is w.a.p.

In the next two propositions $I$ will denote a minimal ideal in the enveloping semigroup of the flow $(X, T)$. Note that $(I, T)$ is a minimal subflow of $(E(X), T)$.

Proposition II.4. The flow $(I, T)$ is almost periodic.

Proof. By Theorem I. 2 it is enough to show that each element of $E(I)$ is continuous (as a map from $I$ to $I$ ). Let $\phi \in E(I)$, say $\phi=\lim t_{\alpha}$. This means that $\lim p t_{\alpha}=p \phi \quad(\forall p \in I)$. Without loss of generality let $t_{\alpha} \rightarrow q \in E(X)$. Then by the continuity of $L_{p}$, we have $p \phi=\lim p t_{\alpha}=p q$. This shows that $\phi=R_{q}$, and by the weak almost periodicity of $(X, T)$, the map $R_{q}$ is a continuous.

Proposition II.5. With the notation as above, we have the following.

(1) The minimal right ideal I has a unique idempotent $u$.

(2) The ideal I is a compact topological group with identity $u$.

(3) The ideal I is also a minimal left ideal.

(4) The ideal $I$ is the only minimal right ideal in $E(X)$.

(5) Let $p \in E(X)$; then $p u=u p$. 
Proof. (1) Any two idempotents in $I$ are proximal (see [4]) and since $(I, T)$ is almost periodic, it cannot have proximal pairs of points.

(2) This follows from (1) and Proposition I.1.

(3 and 4) Since the right multiplication $R_{p}(p \in E(X))$ is continuous, the left $T$ action $(p, t) \rightarrow t p$ also yields a flow on $E(X)$. Now repeating the same arguments, it follows that a minimal left ideal $L$ in $E(X)$ is also a compact topological group with identity, say $v$.

Consider the set $I L=\{p q \mid p \in I, q \in L\}$ Note that $I L \subseteq I \cap L$. Now $u v \in I L \subseteq I$ and since $I$ is a group with identity $u$, there exists a $q \in I$ such that $q(u v)=u$. Thus,

$$
u v=(q u v) v=q u v^{2}=q u v=u .
$$

Hence $u \in L$. Since $u$ is an idempotent and $L$ is a group, $u=v$. Now if $p \in I, p=p u=p v \in L$, and similarly if $q \in L$, then $q=v q=u q \in I$, thus $I=L$. This argument shows that any minimal right ideal coinsides with any given minimal left ideal. This proves (3) and (4).

(5) Let $p \in E(X)$; then by (3) $p u \in I$ and $u p \in I$. Since $u$ is the identity in $I, p u=u(p u)=(u p) u=u p$.

Corollary II.6. Let $(X, T)$ be a w.a.p. flow and I be the unique minimal ideal in $E(X)$. Then $I=E(X)$ iff $x u=x, \forall x \in X$.

Proof. If $I=E(X)$, then $E(X)$ is a group with idempotents $u$ and $e$-the identity of $T$, thus $u=e$. Conversely, if $x u=x, \forall x \in X$, then for any $p \in E(X)$ we have $x p=x u p, \forall x \in X$. Thus $p=u p \in I$.

Remark II.7. Notice that $(X, I)$ is a flow. Set $R=\{(x, y) \mid x I=y I\}$. Then $R$ is a closed equivalence relation and $(x, y) \in R$ iff $\overline{\operatorname{Orb}(x)}$ and $\overline{\operatorname{Orb}(y)}$ contain the same minimal set, namely $x I$. Also it follows that $X I=\{x p \mid p \in I$, $x \in X\}$ is the union of minimal sets and $(X u, T)$ is almost periodic, where $X u=\{x u \mid x \in X\}$. Furthermore, the proximal relation on $X$ is a closed equivalence relation.

Proposition II.8. Let $(X, T)$ be a w.a.p. flow.

(1) If $(X, T)$ is minimal, then $(X, T)$ is almost periodic.

(2) If $(X, T)$ is distal, then $(X, T)$ is almost periodic.

Proof. (1) Since $(X, T)$ is minimal, $X=x I, \forall x \in X$. Fix any $x \in X$ and let $y=x u$; then

$$
(y \cdot t) u=(y u) \cdot t=\left(x u^{2}\right) \cdot t=y \cdot t
$$

Now since $u$ is continuous and the orbit of $y$ is dense in $X, x u=x, \forall x \in X$. Hence by Corollary II.6, $(X, T)$ is almost periodic.

(2) Let $(X, T)$ be distal; then $E(X)$ is a group. Hence $u=e$. Again by Corollary II. 6 this implies that $(X, T)$ is almost periodic.

Proposition I1.9. Let $(X, T)$ be a w.a.p. flow. If there exists a $p \in E(X)$ such that $t p=p($ or $p t=p), \forall t \in T$, then

(a) $p=u, I=\{u\}$, and all minimal sets are singleton.

(b) If $\left\{x_{0}\right\}$ is a minimal set in $\overline{\operatorname{Orb}(x)}$ then $x u=x_{0}$. 
Proof. Since $(X, T)$ is w.a.p. and $t p=p, \forall t \in T$, it follows that $\{p\}$ is a minimal left (right) ideal. Thus (a) follows from Proposition II.5. Now (b) is a simple consequence of (a).

Proposition II.10. Let $(X, T)$ be a w.a.p. flow with a unique minimal set. Then $(X, T)$ is uniquely ergodic.

Proof. Let $R=\{(x, y) \mid x u=y u\}$. Then $R$ is a closed invariant equivalence relation on $X$. Since the map $p \rightarrow\left(x_{0} p\right) R$ from $I$ to $(X / R)$, is an epimorphism, the flow $(X / R, T)$ is almost periodic. Furthermore, since $(X, T)$ has only one minimal set, the flow $(X / R, T)$ is minimal.

For $f \in C(X), \phi(f)=\left({ }_{u} f\right)$ defines an element of $C(X / R)$. Thus $\phi: C(X)$ $\rightarrow C(X / R)$ is an algebra homomorphism with $\phi\left({ }_{t} f\right)=\left({ }_{t} \phi\right)(f), \forall t \in T$. Since $(X / R, T)$ is minimal and almost periodic, it has a unique $T$ invariant Borel probability measure $m$. Define $\mu(f)=m(\phi(f))$. Clearly $\mu$ defines a $T$ invariant Borel probability measure on $X$. Let $\nu$ be another $T$ invariant Borel probability measure on $X$. Let $f \in C(X)$ and $t_{\alpha}$ be any net such that $t_{\alpha} \rightarrow u$. Note that, since $f$ is w.a.p., $\left({ }_{t_{\alpha}} f\right) \rightarrow\left({ }_{u} f\right)$ weakly. Hence $\nu\left({ }_{u} f\right)=$ $\lim \nu\left({ }_{t_{n}} f\right)=\nu(f)$. Thus $\nu$ defines a $T$ invariant Borel probability on $(X / R)$. Since $(X / R, T)$ is uniquely ergodic, we have $\nu(f)=\nu\left({ }_{u} f\right)=m\left({ }_{u} f\right)=\mu(f)$, $\forall f \in C(X)$. Thus $(X, T)$ is uniquely ergodic.

Now we turn to a different question. Given a w.a.p. flow $(X, T)$, we would like to know under what conditions the map $t \rightarrow \pi_{t}$ from $T$ into $E(X)$ is an imbedding. This question is of particular importance in studying the notion of rigidity in ergodic theory, (§III.B and §IV). Let $\tau$ denote the topology on $T$ and $\omega$ denote the induced topology on $T$ as a subset of $E(X)$. Clearly $\omega \subseteq \tau$. We want to know when $\omega$ equals $\tau$. First we introduce some notation.

Let $\beta T$ be the Stone-Čech compactification of $T$ (see [4]). The space $\beta T$ has a natural right $T$ action and in fact the flow $(\beta T, T)$ is the universal point transitive flow for the group $T$. Since the flow $(E(X), T)$ is point transitive, there is a canonical factor map $\rho: \beta T \rightarrow E(X)$. Points of $\beta T$ will be thought of as ultrafilters on $T$. Note that given any $\omega$-neighborhood $W$ of $p, p \in \beta T$, we have $\{t \in T \mid t \in W\} \in p$. Also, if $p, q \in \beta T$, then $p q=\{A \subseteq T \mid A p \in q\}$, where $A p=\left\{t \in T \mid A t^{-1} \in p\right\}$.

Let $N(\tau)=\{p \in \beta T \mid(T-K) \in p$ for all $\tau$-compact subsets $K\}$. (In this paper - will be used to denote the set theoretic difference between two sets.) Note that $N(\tau)$ is the set of ultrafilters which converge to infinity. Furthermore, $N(\tau)$ is a closed invariant right ideal in $\beta T$. The following facts are easy to verify.

(i) If $p \in N(\tau)$ and $p^{-1}=\left\{u^{-1} \mid u \in p\right\}$ then $p^{-1} \in N(\tau)$.

(ii) If $p \in N(\tau)$ then $p p^{-1} \in N(\tau)$.

(iii) Let $p, q \in \beta T$, then $\rho(p q)=\rho(p) \rho(q)$. 
Let $G=\{p \in E(X) \mid p$ is invertible, i.e., there exists a $q \in E(X)$ such that $p q=q p=e\}$. Clearly $T \subseteq G$.

Theorem II.11. Let $(X, T)$ be a w.a.p. flow. Then the following statements are equivalent.

(a) The $\tau$ and $\omega$ topologies on $T$ are the same.

(b) If $p \in N(\tau)$ then $\rho(p) \neq e$.

Furthermore either of the above implies the following.

(c) The sets $T$ and $G$ are the same.

Proof. Let $\eta_{e}(\tau)=\{V \subseteq T \mid V$ is a $\tau$ neighborhood of $e\}$ and $n_{e}(\omega)=\{U \subseteq$ $E(X) \mid U$ is an $\omega$ neighborhood of $e\}$.

(a) $\Rightarrow(\mathrm{b})$ : Suppose there exists a $p \in N(\tau)$ such that $\rho(p)=e$. Since $p \in N(\tau)$, there exists a $\tau$-compact $V \in \eta_{e}(\tau)$ such that $(T-V) \in p$. Now (a) implies that there exists $N \in \eta_{e}(\omega)$ such that $N \cap T \subseteq V$, hence $N \cap T \notin p$. Thus $\rho(p) \neq e$. This is a contradiction.

(b) $\Rightarrow(\mathrm{a})$ : Suppose (a) is false. Then there exists a $\tau$-compact $V \in \eta_{e}(\tau)$ such that $(N \cap T) \cap(T-V)$ is nonempty (where $\left.N \in \eta_{e}(\omega)\right)$. Let $p$ be the ultrafilter containing the filter base $\left\{(T-V) \cap N \mid N \in \eta_{e}(\omega)\right\}$. Since $V$ is $\tau$-compact and $V \notin p, p \in N(\tau)$. Now it follows that $\rho(p)=e$, which is a contradiction.

(b) $\Rightarrow(\mathrm{c})$ : Suppose (c) is false. Since $\rho$ is onto, there exists a $p \in \beta T$ such that $\rho(p) \in(G-T)$. Note that $p \in N(\tau)$ (if not, then there exists a $\tau$ compact subset $K \subseteq T$ such that $K \in p$. This implies that $\rho(p) \in \rho(K) \subseteq T$, which is a contradiction). Also observe that, since $\rho(p)^{-1}$ exists, we must have $\rho\left(p^{-1}\right)=\rho(p)^{-1}$, where $p^{-1}=\left\{U^{-1} \mid U \in p\right\}$.

Let $q=p p^{-1} \in N(\tau)$. Then $\rho(q)=\rho(p) \rho(p)^{-1}=e$. This contradicts (b).

Remark II.12. (i) Note that in the proof of Theorem II.11, local compactness of the topology $\tau$ was crucial.

(ii) We will show that under the additional assumption of metrizability of $E(X)$, condition (c) implies (a). To do this we first prove a couple of lemmas.

Lemma II.13. Let $(X, T)$ be a w.a.p. flow and $(E(X), \omega)$ be metrizable. Then the set $G$ is a residual subset of $E(X)$.

Proof. Let $\left\{V_{n} \in \eta_{e}(\omega) \mid n \in N\right\}$ be a neighborhood base at $e$ such that $V_{1} \supseteq$ $V_{2} \supseteq V_{3} \cdots$. Set $G_{n}=\left\{p \in E(X) \mid p t \in V_{n}\right.$ for some $t \in T$ and $t^{\prime} p \in V_{n}$ for some $\left.t^{\prime} \in T\right\}$. Clearly each $G_{n}$ is an open subset. Furthermore $T \subseteq G_{n}$, $\forall n \in N$. Thus each $G_{n}$ is dense. Now the proof follows from the observation that $G=\bigcap\left\{p \in G_{n} \mid n \in N\right\}$.

Lemma II.14. Let $(X, T)$ be a w.a.p. flow and let $(E(X), T)$ be metrizable. Consider the map $\pi(p, q)=p q, p, q \in E(X)$. Let $q \in E(X)$; then $\pi$ is continuous at $(e, q)$.

Proof. Using a general result on joint continuity (see exercise (20), p. 83 of [2]) it follows that for each $q \in E(X)$, there exists a residual set $F=F(q) \subseteq E(X)$ 
such that if $p \in F$ then $(p, q)$ is a point of continuity of $\pi$. Using Lemma II.13, we can assume without loss of generality that $p \in G$. Let $p_{n} \rightarrow e$. Then $p p_{n} \rightarrow p$, whence $p p_{n} q_{n} \rightarrow p q$. Since $p \in G$, this implies that $p_{n} q_{n} \rightarrow q$.

Proposition II.15. Let $(X, T)$ be a w.a.p. flow and $(E(X), \omega)$ be metrizable. Then conditions (a), (b), and (c) in Theorem II.11 are equivalent.

Proof. We will show that (c) implies (a). Since $T=G$, by Lemma II.13 it follows that $(T, \omega)$ is a residual subset of $E(X)$ and thus a Baire space. Furthermore, Lemma II.14 implies that $(T, \omega)$ is a topological group. Thus by the open mapping theorem, the identity map from $(T, \tau)$ onto $(T, \omega)$ is open. This proves (a).

Remark II.16. When the group $T$ is separable, to analyze various dynamical properties of $(E(X), T)$ one need not assume metrizability of $E(X)$. We can reduce the general case to the metrizable case as follows.

Let $G$ be a countable dense subgroup of $T$ and $f \in C(E(X))$. Define a relation $R$ on $E(X)$ as follows. Let $x, y \in E(X)$; then $x R y$ iff $f(s x t)=$ $f(s y t), \forall s, t \in G$. Then $R$ is a closed invariant equivalence relation on $E(X)$ such that (i) $x R y$ implies that $a x b=a y b, \forall a, b \in E(X)$, and (ii) $E(X) / R$ is metrizable. There is a natural action of $T$ on $E(X) / R$ such that $(E(X) / R, T)$ is a w.a.p. flow with $E(E(X) / R, T) \equiv E(X) / R$. Thus if a certain dynamical property is true for all metrizable enveloping semigroups, then it would be true for all enveloping semigroups.

\section{Applications}

Here we study two specific examples of w.a.p. flows. The first example yields a simple proof of Ryll Nardzewski's theorem [9]. The second example illustrates the importance of w.a.p. flows in ergodic theory.

III.A. The Ryll Nardzewski theorem. Let $T$ be a locally compact, Hausdorff topological group and $C B(T)$ denote the space of bounded continuous functions on $T$. A function $f \in C B(T)$ is called weakly almost periodic iff the set $\left\{{ }_{t} f \mid t \in T\right\}$ has compact closure in the weak topology of $C B(T)$, where ${ }_{t} f(s)-f(s t)$. Let $W(T)$ denote the space of all w.a.p. functions on $T$. Recall that the flow $(\beta T, T)$ is the universal point transitive flow for the group $T$. We will regard $W(T)$ as a subset of $C(\beta T)$. As such, it is a uniformly closed $T$ invariant subalgebra of $C(\beta T)$. With every such subalgebra one associates a point transitive flow $(|W|, T)$, (where $|W|$ is the maximal ideal space of algebra $W(T)$ ) so that $W(T) \equiv C(|W|)$ (see [4] for details).

Proposition III.A.1. The flow $(|W|), T)$ is weakly almost periodic.

Proof. Since $C(|W|) \equiv W(T)$, the proof follows from the definition of weak almost periodicity.

Theorem III.A.2. The linear space $W(T)$ has a left and a right invariant mean. Proof. Since $(|W|, T)$ is w.a.p. and point transitive, it is uniquely ergodic. Let $m$ be the unique $T$ invariant probability measure on $|W|$. Since any 
$f \in W(T)$ can be thought of as a continuous map on $|W|, m$ defines a mean on $W(T)$. Furthermore, from Proposition II.10 it follows that

(i) $m\left({ }_{u} f\right)=m(f)$ and

(ii) $m\left({ }_{p} f\right)=m(f)=m\left(f_{p}\right)$, where $p \in I$ and $I$ is the minimal ideal in $E(|W|)$.

Thus $m$ is a left and right invariant mean on $W(T)$.

Now consider the following subspaces of $C B(T)$.

(i) $A P(T)=\left\{f \in C B(T) \mid f\right.$ is almost periodic; i.e., the set $\left({ }_{t} f \mid t \in T\right)$ has compact closure in the norm topology .

(ii) $W_{0}(T)=\left\{f \in W(T) \mid\left({ }_{u} f\right)=0\right\}$.

(iii) $C_{0}(T)=\{f \in C B(T) \mid f$ vanishes at infinity $\}$.

Note that $A P(T)$ and $W_{0}(T)$ are closed subspaces of $W(T)$; in fact, we have the following theorem.

Proposition III.A.3. With the above notation we have $W(T)=A P(T) \oplus W_{0}(T)$. Proof. Let us write $f=\left({ }_{u} f\right)+\left(f-{ }_{u} f\right)$. Note that, ${ }_{u} f \in A P(T)$ and ${ }_{u}\left(f-{ }_{u} f\right)=0$. Thus $f-{ }_{u} f \in W_{0}(T)$. On the other hand, if $g \in A P(T) \cap W_{0}(T)$ then $g=\left({ }_{u} g\right)$ and ${ }_{u} \bar{g}=0$, hence $g=0$. This completes the proof.

Remark III.A.4. In general, $C_{0}(T) \subseteq W_{0}(T)$, but these two subspaces may not be the same. The group $T$ is said to be minimally weakly almost periodic if $C_{0}(T)=W_{0}(T)$. Using the techniques developed here, we will show (in $\S I V$ ) that the group $\operatorname{SL}(2, R)$ is minimally weakly almost periodic.

III.B. W.a.p. flows and ergodic theory. Let $(\Omega, T, m)$ be a flow with a $T$ invariant probability measure $m$. Here the action of $T$ may only be jointly measurable. We will now associate a w.a.p. system to the flow $(\Omega, T, m)$.

Let $H=L^{2}(\Omega, m), B(H)$ be the set of bounded operators on $H$, and $S=\{L \in B(H) \mid\|L\| \leq 1\}$. The set $S$ is equipped with the weak operator topology. Thus $S$ is a compact space.

Let $U_{t} f(\omega)=f(\omega \cdot t), t \in T, \omega \in \Omega$, and $f \in H$. Set $\mathscr{E}=\overline{\left\{U_{t} \mid t \in T\right\}} \subseteq$ $S$, where the closure is again with respect to the weak operator topology. Thus $\mathscr{E}$ is a compact space which is also a semigroup. Also note that the left and right multiplications in $\mathscr{E}$ (i.e., the maps $L_{A}(B)=A B$ and $R_{A}(B)=B A$, $A, B \in \mathscr{E}$ ) are separately continuous. Furthermore, there is a natural right $T$ action on $\mathscr{E},\left\{(A, t) \rightarrow A \cdot U_{t}, A \in \mathscr{E}, t \in T\right\}$ and by the joint continuity theorem of [3], $(\mathscr{E}, T)$ is in fact a flow.

Henceforth we will identify $t \in T$ with $U_{t} \in \mathscr{E}$ as well as with the map $\pi_{t} \in E(\mathscr{E})$, where $\pi_{t}(A)=A U_{t}$.

Proposition III.B.1. The flow $(\mathscr{E}, T)$ is weakly almost periodic.

Proof. Let $\phi \in E(\mathscr{E})$ and let $t_{\alpha} \rightarrow \phi$. Identifying $t$ with $U_{t}$, we can assume without loss of generality that $t_{\alpha r} \rightarrow B \in \mathscr{E}$. This implies that for any $A \in \mathscr{E}$ we have $A \phi=\lim A t_{\alpha}=A B$. Thus $\phi=R_{B}$ on $\mathscr{E}$. Since $R_{B}$ is continuous, so is $\phi$. Now Proposition II. 2 completes the proof. 
This proof shows that $E(\mathscr{E})$ can be identified with itself. Thus we will think of the unique minimal ideal $I$ of $E(\mathscr{E})$ as a compact group inside the set $\mathscr{E}$. Let $u$ be the idempotent and $P$ and $C$ denote the projections on $T$ invariant functions and constant functions, respectively. Also, if $A \in B(H)$, the adjoint of $A$ will be denoted by $A^{*}$. It is each to verify that $u^{*}=u$, $I^{*}=\left\{A^{*} \mid A \in I\right\}=I, \mathscr{E}^{*}=\mathscr{E}$, and the map $A \rightarrow A^{*}$ is continuous.

Let $\lambda$ be the normalized Haar measure on $I$ and set

$$
\mu(A)=\lambda(A \cap I), \quad \forall \text { Borel subsets } A \subseteq \mathscr{E} .
$$

Proposition III.B.2. With the notation as above, we have the following.

(a) If $p \in I$ then $p p^{*}=p^{*} p=u$.

(b) Let $B \subseteq \mathscr{E}$ be a Borel set; then

(bi) $\mu\left(B^{*}\right)=\mu(B)$ and

(bii) $\mu\left(\left(L_{g}\right)^{-1}(B)\right)=\mu(B)=\mu\left(\left(R_{g}\right)^{-1}(B)\right), \forall g \in \mathscr{E}$.

Proof. (a) Let $p=\lim t_{\alpha}, t_{\alpha} \in T$; then $u t_{\alpha} \rightarrow u p=p$ and $u\left(t_{\alpha}\right)^{-1}=\left(t_{\alpha}\right)^{-1} u=$ $\left(t_{\alpha x}\right)^{*} u=\left(u t_{\alpha x}\right)^{*} \rightarrow p^{*}$. Thus,

$$
u=\lim \left(u\left(t_{\alpha}\right)^{-1}\right)\left(u t_{\alpha}\right)=\lim \left(u\left(t_{\alpha}\right)^{-1}\right) \lim \left(u t_{\alpha}\right)=p p^{*} .
$$

Similarly, $p^{*} p=u$.

(b)(i) By (a) if $p \in I$, then the inverse of $p$ is $p^{*}$. Hence for all Borel subsets $B$ of $I, \lambda\left(B^{*}\right)=\lambda(B)$. Now if $B \subseteq \mathscr{E}$ then

$$
\mu(B)=\lambda(B \cap I)=\lambda(B \cap I)^{*}=\lambda\left(B^{*} \cap I\right)=\mu\left(B^{*}\right) .
$$

(b)(ii) First let $t \in T$; then we have

$$
\mu(t B)=\lambda(t B \cap I)=\lambda(t B \cap t I)=\lambda(t(B \cap I))=\lambda(B \cap I) .
$$

Thus $\mu(t B)=\mu(B)$. Similarly, $\mu(B t)=\mu(B)$. Now let $g \in \mathscr{E}$, and set $p=(u g)^{*}=u g^{*} \in I$. We claim that $\left(L_{g}\right)(B) \cap I=p(B \cap I)$. Assuming this, we get $\mu\left(\left(L_{g}\right)^{-1}(B)\right)=\lambda(p(B \cap I))=\lambda(B \cap I)=\mu(B)$. Now we only have to prove this claim. Let $x \in\left(L_{g}\right)^{-1}(B) \cap I$; then $g x \in B$. Therefore $p g x=p g u x=p p^{*} x=u x=x$. Thus $x \in p(B \cap I)$. Conversely, if $x=p y$ for some $y \in B \cap I$, then $g x=g p y=g u p y=p^{*} p y=u y=y$, hence $x \in\left(L_{g}\right)^{-1}(B) \cap I$. The proof of invariance of $\mu$ under $R_{g}$ is similar.

Proposition III.B.3. With the notation as above, we have $P=\int g d \mu(g)$. Thus the system is ergodic iff $C=\int g d \mu(g)$.

Proof. Let $Q=\int g d \mu(g)$. This integral exists in the sense that

$$
\langle Q a, b\rangle=\int_{\varepsilon}\langle g a, b\rangle d \mu(g)=\int_{I}\langle g a, b\rangle d \mu(g), \quad \forall a, b \in H .
$$


Now (bi) and (bii) of the previous proposition imply that $Q=Q^{*}$ and $Q g=$ $g Q=Q, \forall g \in \mathscr{E}$. Furthermore we have

$$
\begin{aligned}
\left\langle Q^{2} a, b\right\rangle & =\langle Q a, Q b\rangle=\int\langle g a, Q b\rangle d \mu(g)=\int\langle Q g a, b\rangle d \mu(g) \\
& =\int\langle Q a, b\rangle d \mu(g)=\langle Q a, b\rangle .
\end{aligned}
$$

Thus $Q^{2}=Q$. Since $Q t=Q, \forall t \in T$, we have Range $Q \subseteq$ Range $P$. Conversely, if $a \in H$ is such that $t a=a, \forall t \in T$, then $g a=a, \forall g \in \mathscr{E}$. Hence $\langle Q a, b\rangle=\int\langle g a, b\rangle d u(g)=\langle a, b\rangle$. Thus Range $P \subseteq \operatorname{Range} Q$. This shows that $P=Q$.

Lemma III.B.4. The idempotent $u$ is the identity operator on any finitedimensional invariant subspace.

Proof. Let $F \subseteq H$ be a finite-dimensional invariant subspace. Let $t_{\alpha} \rightarrow u$, $t_{\alpha} \in T$. Since $F$ is finite-dimensional, weak convergence of bounded operators on $F$ implies strong convergence. Thus $\left\|U_{t} a-u a\right\| \rightarrow 0, \forall a \in F$. Hence $\|u a\|=\|a\|, \forall a \in F$. The result now follows since $u=u^{*}=u^{2}$.

The system $(\Omega, T, m)$ is said to be weakly mixing if the space of constant functions is the only finite-dimensional subspace of $H$ that is invariant under the unitary representation $U_{t}$. The system is said to have discrete spectrum if $H$ is the Hilbert space direct sum of finite-dimensional invariant subspaces of $U_{t}$.

Proposition III.B.5. The following statements are equivalent.

(1) The system $(\Omega, T, m)$ is weakly mixing.

(2) $u=C$.

(3) $C \in \mathscr{E}$.

Proof. $(1) \Rightarrow(2)$ : Since $p u=p \quad(p \in I)$ the compact group $I$ acts on the image of $u$. Hence $u(H)$ must be the space of constants. Thus $u=C$.

$(2) \Rightarrow(3)$ : This is trivial.

(3) $\Rightarrow(1)$ : Since $C=C u=u C \in I$ and $C$ is a projection, we conclude that $C=u ;(1)$ now follows from Lemma III.B.4.

Proposition III.B.6. The following statements are equivalent.

(1) The system has discrete spectrum.

(2) $u=e$, where $e$ is the identity operator.

(3) $\mathscr{E}=I$.

Proof.

(1) $\Rightarrow(2)$ : This follows from Lemma III.B.4.

(2) $\Rightarrow(3):$ If $p \in \mathscr{E}$ then $p=e p=u p \in I$, whence $\mathscr{E}=I$.

$(3) \Rightarrow(1)$ : Since $\mathscr{E}$ is a compact topological group, (1) follows from the Peter Weyl theorem. 
Remark III.B.7. (a) A vector $a \in H$ is called a compact vector iff the set $\left\{U_{t} a \mid t \in T\right\}$ has compact closure in the norm topology. It is not hard to show that all vectors in $H$ are compact iff $(\Omega, T, m)$ has discrete spectrum.

(b) If we let $A=\left\{a \in H \cap L^{\infty}(\Omega, m) \mid a\right.$ is compact $\}$, then $A$ is a $T$ invariant subalgebra (more precisely it is a $L^{\infty}(\Omega)$ module; see [13]). Hence there exists a factor dynamical system $\left(Y, T, m^{\prime}\right)$ such that $\bar{A}$ is the pullback of $L^{2}\left(Y, m^{\prime}\right)$ under the factor map. This factor is the maximal factor with discrete spectrum and $(\Omega, T, m)$ is its relative weak mixing extension.

Now we study a generalization of the notion of mild mixing introduced by H. Furstenberg and B. Weiss. Since $(\mathscr{E}, T)$ is a point transitive flow, there is a canonical factor map $\rho: \beta T \rightarrow \mathscr{E}$. We recall the notion of convergence associated with an ultrafilter.

(i) Given $p \in \beta T$ and $a \in H$, we write $\lim _{t \in p} U_{t} a=b$ or $\rho(p)=b$ iff $\left\{t \mid\left\|U_{t} a-b\right\|<\varepsilon\right\} \in p, \forall \varepsilon>0$.

(ii) Given $p \in \beta T$ and $A \in \mathscr{E}$, we write $\lim _{t \in p} U_{t}=A$ iff for any $a, b \in H$ and $\varepsilon>0$, we have $\left\{t||\left\langle U_{t} a, b\right\rangle-\langle A a, b\rangle \mid<\varepsilon\right\} \in p$.

Since the weak and strong topologies on $\left\{U_{t} \mid t \in T\right\}$ coincide, if $A \in\left\{U_{t} \mid t \in\right.$ $T\}$ then $\lim _{t \in p} U_{t}=A$ implies that $\rho(p) a=A a, \forall a \in H$. Notice that the notation in $\S$ II and proofs of Theorem II.11 through Theorem II. 15 are also valid after replacing $(E(X), T)$ by $(\mathscr{E}, T)$. The flow $(\Omega, T, m)$ is rigid iff there exists a $p \in N(\tau)$ such that $\rho(p)=e$. Note that this means that $\lim _{t \in p} U_{t}=e$. The flow $(\Omega, T, m)$ is mild mixing if it has no nontrivial rigid factors. Now Theorem II.11 implies the following.

Proposition III.B.8. The following statements are equivalent.

(a) The system $(\Omega, T, m)$ is not rigid.

(b) The $\tau$ and $\omega$ topologies on $T$ are the same.

Furthermore, if $(\mathscr{E}, T)$ is metrizable (in particular if $(\Omega, m)$ is a standard Borel space) then the above statements are also equivalent to the following.

(c) The sets $T$ and $G$ are the same.

A vector $a \in H$ is called rigid iff there exists $p \in N(\tau)$ such that $\rho(p)(t a)=$ $t a, \forall t \in T$. Note that for abelian $T, \rho(p) a=a$ implies that $a$ is rigid.

Proposition III.B.9. The system $(\Omega, T, m)$ is mild mixing iff the constants are the only rigid vectors.

Proof. Let $(\Omega, T, m)$ be mild mixing and $a \in H$ be a rigid vector. Let $\left(Y, T, m^{\prime}\right)$ be a factor of $(\Omega, T, m)$ such that $L^{2}\left(Y, m^{\prime}\right)$ is isomorphic to the algebra generated by $\{t a \mid t \in T\}$. Since $a$ is rigid, there exists a $p \in \beta T$ such that $\rho(p)(t a)=t a, \forall t \in T$; thus $\rho(p)(b)=b, \forall b \in L^{2}\left(Y, m^{\prime}\right)$. Thus $\left(Y, T, m^{\prime}\right)$ is a rigid factor. Hence $Y$ is a point space. This implies that $a$ is a constant function.

Conversely, let $\left(Y, T, m^{\prime}\right)$ be a rigid factor of $(\Omega, T, m)$. Then there exists $p \in N(\tau)$ such that, $\rho(p) b=b, \forall b \in L^{2}\left(Y, m^{\prime}\right)$. Thus for any $g \in L^{2}\left(Y, m^{\prime}\right)$, 
$\rho(p)(t g)=t g, \forall t \in T$. Hence $g$ must be a constant function and $Y$ must be a point space.

Proposition III.B.10. Let the group $T$ be abelian. Then the following statements are equivalent.

(a) The system $(\Omega, T, m)$ is mild mixing.

(b) If for some $p \in N(\tau),\|\rho(p) g\|=\|g\|$, then $g$ is a constant function.

(c) For all nonconstant vectors $a \in H$, we have

$$
\operatorname{Sup}_{p \in N(\tau)}|\langle\rho(p) a, a\rangle|<\|a\|^{2} .
$$

Proof. Given $p \in \beta T$, recall that $p^{-1}=\left\{U^{-1} \mid U \in p\right\}$. Note that in this case (i.e., $E(X) \equiv \mathscr{E}) \rho\left(p^{-1}\right)=\rho(p)^{*}$.

(a) $\Rightarrow($ b): Suppose there exist $p \in N(\tau)$ and $a \in H$ such that $\|\rho(p) a\|=$ $\|a\|$. Set $q=p^{-1} p \in N(\tau)$; then

$$
\|\rho(q) a-a\|^{2}=\|\rho(q) a\|^{2}+\|a\|^{2}-\langle\rho(q) a, a\rangle-\left\langle\rho(q)^{*} a, a\right\rangle=0 .
$$

Thus $a$ is a rigid vector and hence must be constant.

(b) $\Rightarrow$ (c): If (c) is false then since $N(\tau)$ is closed there exist $p \in N(\tau)$ and a nonconstant vector $g \in H$ such that $|\langle\rho(p) g, g\rangle|=\|g\|^{2}$. Again let $q=p^{-1} p$. Then as before it is easy to check that $\rho(q) g=g$, whence $g$ is a constant vector, which is a contradiction.

(c) $\Rightarrow(a)$ : This is easy to see.

Finally, we formulate the notion of strong mixing as follows. The system $(\Omega, T, m)$ is called strongly mixing iff $\rho(p)=u, \forall p \in N(\tau)$, where $u$ is the unique idempotent in $I \subseteq \mathscr{E}$. Note that $u \neq e$ unless $(\Omega, T, m)$ is trivial.

Proposition III.B.11. The system $(\Omega, T, m)$ is strongly mixing iff $(\mathscr{E}$, $\omega)$ is the one point compactification of $(T, \tau)$.

Proof. Let $(\Omega, T, m)$ be strongly mixing. From the definition it follows that any weak limit of a net $U_{t_{0}}$ (where $t_{\alpha}$ tends to infinity) must be $u$. Thus $\mathscr{E}=\left\{U_{1} \mid t \in T\right\} \cup\{u\}$ and $u=C$. Also, it is clear that strong mixing implies mild mixing. Hence by Proposition III.B.8 the map $t \rightarrow U_{t}:(T, \tau) \rightarrow(T, \omega)$ is an imbedding, whence $\mathscr{E}$ is the one-point compactification of $(T, \tau)$.

Conversely, if $\mathscr{E}=\left\{U_{1} \mid t \in T\right\} \cup\{u\}$ and $T$ is open in $\mathscr{E}$, then it follows that any net $t_{c r}$ which lies outside a $\tau$-compact set eventually converges to $u$ weakly. This means that $\rho(p)=u, \forall p \in N(\tau)$.

\section{W.A.P. FLOWS OF SEMISIMPLE LIE GROUPS}

In this section we show that any w.a.p. flow $(X, T)$, where $T$ is a semisimple Lie group with finite center and no compact factors, has a certain property (which will be defined shortly). In particular, this will yield a theorem of $\mathrm{W}$. Veech and also the ergodicity theorem of $\mathrm{C}$. Moore.

Let $(X, T)$ be a point transitive w.a.p. flow. This flow is said to have property (A), if (i) the unique minimal set in $(X, T)$ is singleton, say $\{m\}$, and (ii) for any $x \in X$ we have $\overline{\operatorname{Orb}(x)}=\operatorname{Orb}(x) \cup\{m\}$. 
Theorem IV.1. Let $(X, T)$ be a point transitive, weakly almost periodic flow. Let $T$ be a semisimple Lie group with finite center and no compact factors. Then $(X, T)$ has property $(\mathrm{A})$.

Remark IV.2. Let $T$ be a semisimple Lie group and $T=K A N^{+}$be the Iwasawa decomposition. Let $N^{-}$denote the "opposite" nilpotent subgroup. Consider the following condition.

Condition (*). If $a_{n} \in A$ and $a_{n} \rightarrow \infty$, then either (a) or (b) of the following must hold.

(a) $a_{n} t\left(a_{n}\right)^{-1} \rightarrow e, \forall t \in N^{+}$and $\left(a_{n}\right)^{-1} t a_{n} \rightarrow e, \forall t \in N^{-}$.

(b) $a_{n} t\left(a_{n}\right)^{-1} \rightarrow e, \forall t \in N^{-}$and $\left(a_{n}\right)^{-1} t a_{n} \rightarrow e, \forall t \in N^{+}$.

We will first prove Theorem IV.1 for semisimple Lie groups satisfying condition $(*)$. Note that any semisimple Lie group with real rank 1 (in particular $\mathrm{SL}(2, R))$ satisfies this condition. This proof will be generalized to higher rank groups using some standard facts of Lie theory.

Proof of Theorem IV.1 for groups satisfying condition $(*)$. First note that without loss of generality, we can assume that the $T$ action on $X$ is effective (i.e., if $x \cdot t=x, \forall x \in X$, then $t=e)$. Recall that $G=\{p \in E(X) \mid$ there exists $q \in E(X)$ such that $x(q p)=x, \forall x \in X\}$. The proof follows from the following series of lemmas.

Lemma IV.3. Let $a_{n} \in A$ and $a_{n} \rightarrow p \in E(X)-T$. Then $p \notin G$.

Proof. Let $t \in N^{+}$(or $t \in N^{-}$depending on the validity of (a) or (b) of condition $(*))$, be such that $t \neq e$ and $a_{n} t\left(a_{n}\right)^{-1} \rightarrow e$. Then by the joint continuity of the left $T$ action, we have

$$
p t=\lim a_{n} t=\lim a_{n} t\left(a_{n}\right)^{-1} a_{n}=p .
$$

Suppose $p \in G$; then $x(q p)=x, \forall x \in X$, for some $q$. Hence $x=x(q p)=$ $x(q p) t=x \cdot t, \forall x \in X$. This implies that $t=e$, which is a contradiction.

Lemma IV.4. With the above notation we have $T=G$.

Proof. Clearly $T \subseteq G$. Let $p \in G$ and suppose that $p \notin T$. Then considering the Cartan decomposition, $T=K A K$, we may suppose that $p=k r l$, where $k, l \in K$ and $r=\lim a_{n}, a_{n} \in A$. Note that $p \notin T$ implies that $r \in E(X)-T$. Since $p \in G$, there exists $q \in E(X)$ such that $x=x(q p), \forall x \in X$. Hence $x=x(q p)=x(q k r l)$, therefore $x l^{-1}=x(q k r)$, whence replacing $x$ by $x l$, $x=x l(q k r), \forall x \in X$. Since $\operatorname{lq} k \in E(X)$, this shows that $r \in G$, which is a contradiction to the previous lemma.

Lemma IV.5. Let $a_{n} \rightarrow p \in E(X)-T, a_{n} \in A$, and $\left(a_{n}\right)^{-1} \rightarrow q \in E(X)$. Then $p q=q p=u$.

Proof. First note that $\bar{A}$ (the closure as a subset of $E(X)$ ) is an abelian subsemigroup. This follows from the separate continuity of both the left and the right multiplication. Thus $p q=q p$. Now we show that $t(p q)=p q, \forall t \in T$. 
Suppose (a) of condition (*) holds (a similar argument can be given if (b) holds). Note that

$$
t p=\lim t a_{n}=\lim a_{n}\left(\left(a_{n}\right)^{-1} t a_{n}\right)=p, \quad \forall t \in N^{-}
$$

and

$$
t q=\lim t\left(a_{n}\right)^{-1}=\lim \left(a_{n}\right)^{-1}\left(a_{n} t\left(a_{n}\right)^{-1}\right)=q, \quad \forall t \in N^{+} .
$$

Hence $t(p q)=p q, \forall t \in N^{+} \cup N^{-}$. Since $N^{+} \cup N^{-}$generates $T$, the proof follows from Proposition II.9.

Lemma IV.6. Let $p \in E(X)-T$, say $p=k p^{\prime} l$, where $k, l \in K$ and $p^{\prime}=\lim a_{n}$, $a_{n} \in A$. Let $q=l^{-1} q^{\prime} k^{-1}$, where $q^{\prime}=\lim \left(a_{n}\right)^{-1}$. Then $p q=q p=u$.

Proof. Note that $p q=p^{\prime} q^{\prime}=u=q^{\prime} p^{\prime}=q p$, by Lemma IV.5.

Lemma IV.7. Let $p \in E(x)-T$. Then $p=u$.

Proof. First let $p \in \bar{A}-T$, say $p=\lim a_{n}, a_{n} \in A$. Let $t \in N^{-}$(or $N^{+}$ depending on the validity of $(a)$ or $(b)$ of condition $(*))$. Then we have

$$
t p=\lim t a_{n}=\lim a_{n}\left(\left(a_{n}\right)^{-1} t a_{n}\right)=p .
$$

Thus $t^{m} p=p$, for all integers $m$. Again writing $t^{m}=k_{m} a_{m} l_{m}$, where $k_{m}, l_{m} \in K$ and $a_{m} \in A$, and passing to a subsequence if necessary, we let $q=k\left(\lim a_{m}\right) l$, with $k, l \in K$, and $q^{*}=\lim t^{-m}=l^{-1} \lim \left(a_{m}\right)^{-1} k^{-1}$, thus $q q^{*}=u$. Also we have $q p=p$ and $p=q^{*} p$. Hence,

$$
p=q p=q\left(q^{*} p\right)=\left(q q^{*}\right) p=u p=u \text {. }
$$

Now a general $p$ can be written as $p=k r l, k, l \in K$ and $r \in \bar{A}-T$. Thus $p=k u l=u$.

This completes the proof of Theorem IV.1 for groups satisfying condition $(*)$.

Now we extend this result to general semisimple Lie group with finite center and no compact factors. This is done by the standard technique of reducing the proof to the $\operatorname{SL}(2, R)$ case. All such reduction techniques are based on the crucial fact that such groups contain "many" copies of $\operatorname{SL}(2, R)$. We will need to recall some notation and standard facts from [11]. We will do this in the next paragraph. The use of enveloping semigroups techniques will allow us to give simpler and shorter arguments than those of [11] and with less use of Lie group machinery.

Let $T$ be a semisimple Lie group with finite center and no compact factors. Let $\mathscr{T}$ be its Lie algebra. Fix a Cartan decomposition $\mathscr{T}=\mathscr{K}+\mathscr{P}$ and involution $\theta$. Let $\mathscr{A}$ be the maximal abelian subspace of $\mathscr{P}$. Let $\Delta$ be the set of (restricted) roots of the pair $(\mathscr{T}, \mathscr{A})$. Order the roots and let $\Delta^{+}$be the set of positive roots. If $\lambda \in \Delta$, define $\mathscr{T}^{\lambda}=\{X \in \mathscr{T} \mid[H, X]=\lambda(H) X, H \in \mathscr{A}\}$ and set $\mathscr{N}^{ \pm}=\sum_{ \pm \lambda \in \Delta^{+}} \mathscr{T}^{\lambda}$. Let $K, A, N^{ \pm}$be the analytic subgroups of $T$ corresponding to $\mathscr{K}, \mathscr{A}$, and $\mathscr{N}^{ \pm}$. Let $\mathscr{A}^{+}$be the positive Weyl chamber, 
$\mathscr{A}^{+}=\left\{H \in \mathscr{A} \mid \lambda(H)>0, \lambda \in \Delta^{+}\right\}$. Set $A^{+}=\exp \left(\mathscr{A}^{+}\right)$and $\overline{\mathscr{A}^{+}} \subseteq A$, where the closure is in the group $T$, and not as a subset of $E(X)$. Let $B(\cdot, \cdot)$ denote the Killing form. Then for each $\lambda \in \Delta$, there exists $Q_{\lambda} \in \mathscr{A}$ such that $\lambda(\cdot)=B\left(\cdot, Q_{\lambda}\right)$.

If $\alpha=\left(\alpha_{n}\right)$ is a sequence in $T, N_{\alpha}^{+}$shall denote the closed subgroup of $T$ generated by the set $U_{\alpha}^{+}$, where

$$
U_{\alpha}^{+}=\left\{t \mid \underline{\lim } \delta\left(\left(\alpha_{n}\right)^{-1} t \alpha_{n}, e\right)=0\right\} .
$$

Here $\delta$ is a metric on $T$ generating its topology. Where $\alpha$ has the form $\alpha_{n}=b^{n}, n>0$ for a fixed $b \in T$, we shall write $N_{b}^{+}$for $N_{\alpha}^{+}$. Define $N_{b}^{-}=N_{b^{-1}}$ and let $T_{b}$ be the closed subgroup of $T$ generated by $N_{b}^{ \pm}$. We shall need the following facts from Lie theory, (see [11] for proofs).

(F1) If $b \in A$, then $T_{b}$ is a normal subgroup containing $b$. If $b \in A^{+}$then $N_{b}^{+}=N^{+}$and $T_{b}=T$.

(F2) If $\alpha$ is a sequence tending to infinity in $\overline{A^{+}}$, then $\exists b \in \overline{A^{+}}, b \neq e$, such that $N_{a}^{+}=N_{b}^{+}$.

(F3) If $\lambda \in \Delta^{+}$and if $v \in \mathscr{T}^{\lambda}, v \neq 0$, then $v, \theta(v)$ and $Q_{\lambda}$ span a Lie algebra of $\mathscr{T}$ isomorphic to the Lie algebra of $\operatorname{SL}(2, R)$.

(F4) With the notation as above, we have $T=\overline{K A^{+} K}$.

We shall now assume that $T=T_{1} \times T_{2} \times \cdots \times T_{r}$, where each $T_{i}$ is a simple and noncompact Lie group with finite center. We shall identify $T_{l}$ with $\left\{\left(t_{1}, t_{2}, \ldots, t_{r}\right) \mid t_{j}=e_{j}, \forall j \neq l\right\}$. Note that it is enough to prove the theorem for such a $T$ [11]. Finally, let $T_{l}=K_{l} \overline{A_{l}^{+}} K_{l}$ be the modified Cartan decomposition as in (F4).

Lemma IV.8. Let $S$ be a subgroup of $T$ isomorphic to $\operatorname{SL}(2, R)$. Let $a \in A \cap S$, $a \neq e$. Let $u_{S}$ be the unique idempotent in $\bar{S} \subseteq E(X)$. Then $\mu_{S} t=t \mu_{S}=\mu_{S}$, $\forall t \in T_{\alpha}$.

Proof. Since $(X, S)$ is w.a.p. and $S$ is isomorphic to $\operatorname{SL}(2, R)$, we have $\bar{S}-S=\left\{\mu_{S}\right\}$. Since $a \in S$, there is a sequence $n_{i} \rightarrow \infty$ such that $a^{n_{i}} \rightarrow \mu_{S}$ and $a^{-n_{t}} \rightarrow \mu_{S}$. Thus by the argument of Lemma IV.3 we have $\mu_{S} t=\mu_{S}=t \mu_{S}$, $\forall t \in N_{a}^{+} \cup N_{a}^{-}$. Since $N_{a}^{ \pm}$generates $T_{a}$, the proof is complete.

Lemma IV.9. Fix any $l(1 \leq l \leq r)$. Let $\alpha=\left(a_{n}\right) \in \overline{A_{l}^{+}}$. Let $\lim a_{n}=p \in$ $E(X)$. Then $t p=p, \forall t \in T_{l}$.

Proof. By (F2) there exists $b \in \overline{A_{l}^{+}}, b \neq e$, such that $N_{\alpha}^{+}=N_{b}^{+}$. Let $\lambda \in \Delta_{l}^{+}$ be such that $\mathscr{T}_{1}^{\lambda} \subseteq \mathscr{N}_{b}^{+}$. By (F3), there exists $v \in \mathscr{T}^{\lambda} \subseteq \mathscr{N}_{b}^{+}$such that $v \neq 0$ and $v, \theta(v), Q_{\lambda}$ span a Lie algebra isomorphic to the Lie algebra of $\operatorname{SL}(2, R)$. Let $S$ be the Lie subgroup corresponding to this subalgebra. Let $a=\exp \left(Q_{\lambda}\right)$ and $s=\exp (v)$. Note that (i) $a \in A \cap S$ and (ii) $s p=p$ (this is because $v \in \mathscr{T}_{1}^{\lambda} \subseteq \mathscr{N}_{b}^{+}=\mathscr{N}_{\alpha}^{+}$). 
Now Lemma IV.8 implies that $\mu_{S} t=t \mu_{S}=t, \forall t \in T_{\alpha}$. Pick a sequence $k_{i} \rightarrow \infty$ such that $s^{k_{i}} \rightarrow \mu_{S}$. Thus (ii) implies that $\mu_{S} p=p$. Combining this, we get

$$
t p=t\left(\mu_{S} p\right)=\left(t \mu_{S}\right) p=\mu_{S} p=p, \quad \forall t \in T_{a} .
$$

Note that by (F1), $T_{a}$ is a normal subgroup. Since $T_{l}$ is simple, $T_{a} \cap T_{l}=T_{l}$. This completes the proof.

Lemma IV.10. Let $t_{n}=\left(t_{n}^{1}, \ldots, t_{n}^{r}\right) \rightarrow p \in E(X)$, where $t_{n}^{i} \in T_{i}, \quad 1 \leq i \leq r$. Then $p=u$.

Proof. By Proposition II.9 it is enough to show that $t p=p, \forall t \in T$. Let $t_{n}^{i}=k_{n}^{i} a_{n}^{i} l_{n}^{i}$, where $k_{n}^{i}, l_{n}^{i} \in K_{i}$ and $a_{n}^{i} \in \overline{A_{i}^{+}}$. Fix any $i, 1 \leq i \leq r$, and consider $q^{i}=\lim a_{n}^{i}$. Then by the previous lemma $t q^{i}=q^{i}, t \in T_{l}$. Hence $q^{i}=u$. Thus $p^{i}=\lim k_{n}^{i} a_{n}^{i} l_{n}^{i}=k^{i} u l^{i}=u$, where $k^{i}=\lim k_{n}^{i}$ and $l^{i}=\lim l_{n}^{i}$. Thus $p=u$. This completes the proof of Theorem IV.1.

Finally, we note that the following theorems of $W$. Veech and C. Moore which motivated this paper follow at once from Theorem IV.1.

Theorem IV.11 (W. Veech [11]). Let $T$ be a semisimple Lie group with finite center and no compact factors. Then $T$ is minimally weakly almost periodic.

Proof. Apply Theorem IV.1 to the transitive w.a.p. flow $(|W(T)|, T)$. Thus given any $f \in C(|W(T)|) \equiv W(T), \lim _{t \rightarrow \infty} f(t)=m(f)$, where $m$ is the invariant mean on $W(T)$. Thus $C_{0}(T)=W_{0}(T)$.

Theorem IV.12 (C. Moore [8]). Let $(\Omega, T, m)$ be an ergodic flow, where $T$ is a semisimple Lie group with finite center and no compact factors. Then $(\Omega, T, m)$ is strongly mixing.

Proof. The proof follows by applying Theorem IV.1 to the point transitive w.a.p. flow $(\mathscr{E}, T)$.

\section{REFERENCES}

1. J. Auslander, Minimal flows and their extensions, North-Holland, Amsterdam, 1988.

2. N. Bourbaki, Topologie générale, 1948 edition.

3. R. Ellis, Locally compact transformation groups, Duke Math. J. 24 (1957), 119-126.

4. __ Lectures on topological dynamics, Benjamin, New York, 1969.

5. R. Ellis and M. Nerurkar, Enveloping semigroups in ergodic theory, Proc. Special Year in Ergodic Theory, University of Maryland, 1987.

6. A. Grothendieck, Critères de compacité dans les espaces fonctionnels généraux, Amer. J. Math. 74 (1952), 168-186.

7. G. Laison, A semigroup associated with invariant measure on a transformation group, Math. Systems Theory 8 (3) (1975).

8. C. Moore, Ergodicity of flows on homogeneous spaces, Amer. J. Math. 88 (1966), 154-178.

9. C. Ryll Nardzewski, Generalized random ergodic theorem and weakly almost periodic functions, Bull. Acad. Polon. Sci. 10 (1962), 271-175.

10. J. Troillac, Espaces fonctionnels et théorèmes de Namioka, Bull. Soc. Math. France 107 (1979), $127-137$. 
11. W. Veech, Weakly almost periodic functions on semisimple Lie groups, preprint.

12. R. Zimmer, Ergodic theory and semisimple Lie groups, Birkhäuser, Boston, Mass., 1984.

13. __ Extensions of ergodic group actions, Illinois J. Math. 20 (1976), 373-419.

Department of Mathematics, University of Minnesota, Minneapolis, Minnesota 55455

Department of Mathematics, Ohio State University, Columbus, Ohio 43210 